\title{
Correction to: An order theoretic fixed point theorem with application to multivalued variational inequalities with nonsmooth bifunctions
}

\author{
Christoph Tietz®
}

\section{Correction to: J. Fixed Point Theory Appl. (2019) 21:5 https://doi.org/10.1007/s11784-018-0639-x}

In the original paper, we assumed that each countable well-ordered chain $C$ in a poset $D$ equals an increasing sequence $\left(c_{n}\right)$. Unfortunately, there are simple counter-examples. Consider, e.g., the set

$$
C:=\{1-1 / n: n \in \mathbb{N}\} \cup\{1\} .
$$

Although $C$ is a well-ordered subset of $[0,1] \subset \mathbb{R}$, there is no increasing sequence $\left(c_{n}\right) \subset \mathbb{R}$ such that $C=\left\{c_{n}: n \in \mathbb{N}\right\}$.

In consequence, we have to make two corrections to the original paper:

1. The claim "(C1) and (C2) are equivalent" stated after Theorem 2.10 is false, and thus, it should be deleted. Furthermore, Theorem 2.10 only simplifies if $D$ has property $(\mathrm{C} 1)$.

2. The claim " $C$ equals an increasing sequence $\left(c_{n}\right)$ " in the proof of Theorem 2.11 is false. However, the proof will be correct if we do not start with a general chain $C \subset S_{+}=\{x \in D:\{x\} \leq * S(x)\}$, but with an increasing sequence $\left(c_{n}\right) \subset S_{+}$. Then, it is seen exactly like in the original paper that all assumptions of Corollary 0.1 below are satisfied, whence Theorem 2.11 holds true.

To work with posets satisfying Property (C1), we have to add two results to the original paper. To this end, let us recall that a poset $D$ is said to have Property (C1) if, for each nonempty well-ordered subset $C$ of $D$ such that

The original article can be found online at https://doi.org/10.1007/s11784-018-0639-x. 
each increasing sequence in $C$ has an upper bound in $D$, there is an increasing sequence in $C$ that has the same upper bounds as $C$.

First, let us prove the following corollary of Theorem 2.10 of the original paper:

Corollary 0.1. Let $D$ be a poset satisfying Property (C1), and assume that $S: D \rightarrow \mathcal{P}_{\emptyset}(D)$ satisfies the following hypotheses:

(i) The set $S_{+}=\left\{x \in D:\{x\} \leq^{*} S(x)\right\}$ is nonempty.

(ii) For all increasing sequences $\left(c_{n}\right) \subset S_{+}$and $\left(s_{n}\right) \subset D$ such that, for all $n, c_{n} \leq s_{n} \in S\left(c_{n}\right)$, there is an upper bound $s^{\prime} \in S_{+}$of $\left(s_{n}\right)$.

Then $S$ has a maximal fixed point which is also a maximal element of $S_{+}$.

Proof. Let $C \subset S_{+}$be a nonempty well-ordered chain and $s: C \rightarrow D$ an increasing function such that $c \leq s(c) \in S(c)$ for all $c \in C$. Then, for every increasing sequence $\left(s_{n}\right) \subset s(C) \subset D$, let $c_{n}$ be the smallest element of $\left\{c \in C: s(c)=s_{n}\right\}$, such that $\left(c_{n}\right) \subset C \subset S_{+}$is an increasing sequence with $c_{n} \leq s_{n} \in S\left(c_{n}\right)$. From the assumptions, $\left(s_{n}\right)$ has an upper bound $s^{\prime} \in S_{+} \subset D$, and since $s(C)$ is well-ordered and due to $(\mathrm{C} 1)$, we can assume that $\left(s_{n}\right)$ has the same upper bounds as $s(C)$, so that $s^{\prime} \in S_{+}$is an upper bound of $s(C)$. This shows that all assumptions of Theorem 2.10 are satisfied, and thus, $S$ has a maximal fixed point which is also a maximal element of $S_{+}$.

Second, let us give a sufficient condition for Property (C1) to hold:

Proposition 0.2. Let $D$ be a bounded subset of a reflexive ordered Banach space E. Then, D has Property (C1).

Proof. Let $C$ be a nonempty well-ordered chain in $D$. Then, from $[1$, Prop. 1.3.6], we know that there is an increasing sequence $\left(c_{n}\right)$ in $C$ converging weakly to the supremum of $C$. Then, for each upper bound $b$ of $\left(c_{n}\right)$, it follows $\sup C \leq b$, since the set $\{x \in D: x \leq b\}$ is weakly closed. Consequently, $C$ and $\left(c_{n}\right)$ have the same upper bounds, whence $D$ has property (C1).

Since Theorem 2.11 holds true, the rest of the paper is not effected.

Publisher's Note Springer Nature remains neutral with regard to jurisdictional claims in published maps and institutional affiliations.

\section{Reference}

[1] Heikkilä, S., Lakshmikantham, V.: Monotone Iterative Techniques for Discontinuous Nonlinear Differential Equations. Marcel Dekker Inc., New York (1994)

Christoph Tietz

Institut für Mathematik

Martin-Luther-Universität Halle-Wittenberg

06099 Halle (Saale)

Germany

e-mail: christoph.tietz@mathematik.uni-halle.de 